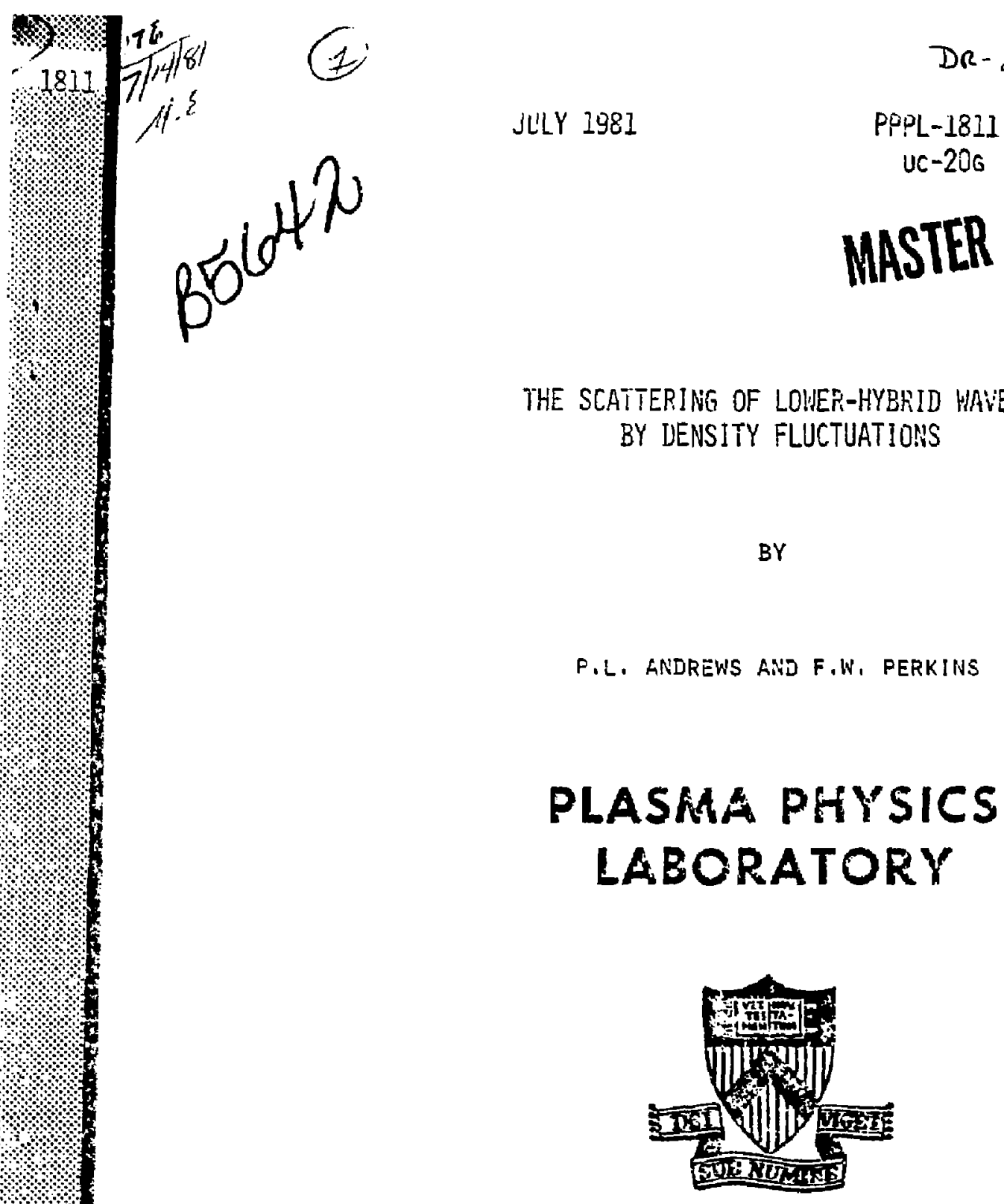

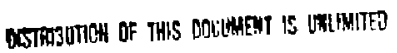 \\ PRIRCEION UNIYERSITY PRINCETON, NEK JERSEY
}

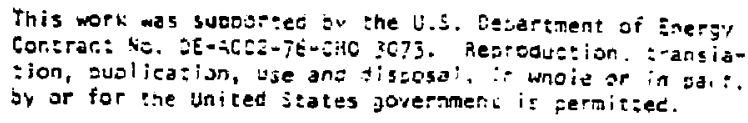




\title{
THE SCATTERING OF LOWER-HYBRID WAVES BY DENSITY FLUCTUATIONS
}

\author{
P. 1. . Indrews and F. W. Perkins \\ Plasma Plysies I.aboratory. Princeton University. \\ Princetun. Neu Jerses 0854
}

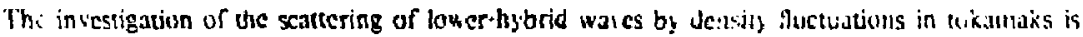

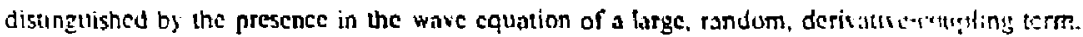
Issunning the fluctuations to be of long warelength ecompared to the incident watte the :ninlanty of the ware callation to the Schrocdinger equation for a garsicle in a randern magnetic field is used In derive a two-way diffusion equation for the wave energ) densily. The diffyrion consun finund disigres with earlier findings and the souree of the discrepancy is pointed out. When the curreet bnundary conditions are imposed this squation can be solved by separation of variahles. Hewever must of the impcrane features of the sulution are appurent witlout dituled algebra.

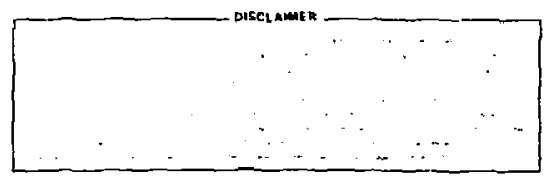




\section{INTROJUUCTION}

The use of lower-hybrid waves as a healing mechanism for confined plasmas has been of great interest for some time as simple propagation theory shows them to have several properties advantagevus 10 their use in tokamaks. "Howcrer. With the recent discovery of drif wave induced large anplitude density flucsuations near the edge of tokamaks ${ }^{23}$, doubt was east an the validity of results derived by dheorics neglecting the sialtering effects of such fluctuations. Sevcral papers have already been written distussing these scattering effects giving roughly equivatent predictions as to the rate of scatter of the incident waves. In one particular regime the wave energy density will obcy a diftusion equation, as hi:s already been derived using a wave-kinetic appronch. ${ }^{+}$lt is the purpose of this paner to derive this cquation in a simple manner, pointing out that some previous results are in error, and to find the incensity and angul.ır spectrum of Ule lower hybrid wave energy emerging from the scattering layer.

In Sec. II ne derive the ware cquation and state the paraneser regime in which we are interested. We then point out the equivalence of this equation to the Schroedinger equation for a cluarged particle in a magnc,ic field. This equivalence is used in Sex. III to derive a diffusion equation for the encrgy density of the wave. Our result disagrecs with some ?revious work and we clarify the origin of the discrepancy in one particular paper. In Sec.IV we show how this diffusion equation can be sulved when the correct boundary condition, are imposed by separation of variables. However an approximate solution for the intensity and angular spectrum of the of the emerging radiation can be found without recourse to complicated algebra and this is done in sectionV. Scction Vi presents our conclusions.

\section{I. THE WIIV FQUITION}

The frequencies of drift waves in tokamaks are far lower Unan lower hybrid frequencies. This permits us to consider propagation of a monochromatic wave in a time-jndependent medium. Experimental investigation ${ }^{2}$ lans shown the density fluctuations to be largely confince th a relativcly thin region near the outside of the cukatnak allowing us to consider the magnetic ficld unifom over the scattering region. 
We thus cheorse a co-ordinate s!stem in which $\dot{B}=B_{1}, \hat{a}$ with $B_{0}$ a constant and $\hat{z}$ the unit vector in the $z$ direction. Then the lower hybrid wate cquation can be wntieti as

$$
\nabla \cdot K \cdot \nabla \phi=0
$$

where the dielsetric tensor $K$ is given by:

$$
K=\left(\begin{array}{ccc}
K_{\perp} & K_{x \nu} & n \\
K_{z \nu}^{*} & K_{\perp} & 0 \\
0 & 0 & K_{\| l}
\end{array}\right)
$$

and we individual components are

$$
K_{\perp}=1-\frac{\omega_{p i}^{2}}{\omega^{2}}+\frac{\omega_{p e}^{2}}{\Omega_{e}^{2}}, \quad K_{\|}=-\frac{\omega_{p e}^{2}}{\omega^{2}}, \quad K_{z y}=-i \frac{\omega_{p s}^{2}}{\omega \Omega_{e}}
$$

The nomenclature is standard: $\omega_{p e}$ and $\omega_{p i}$ are the local electron and ion plasma frequencies respecvively, which here will be functions of position, and $\Omega_{c}$ is due electron gyrofrequency. Vear the edge of the rokatrluk $\left|w_{p c}^{2} / \Omega_{e}^{2}-w_{p l}^{2} / w^{2}\right| \ll 1$ so that $K_{\perp} \approx 1$. In addition the drift waves are atıgned atong the magnetic firld lines with lıtele density variation along $\dot{B}$. Hence we idealize the problem to one where the waves are incident on a two-dimensional scatcring layer of finite thickness. We therefore take the density to be given by

$$
n=n(x, y)=n_{0}[1+\mu(x, y)]
$$

where $n_{0}$ is the spatially constant average density and $\mu$ arises from drif wave density fluctuations. We take $\langle\mu\rangle=0$ the angular brackets denoting an areraging process over realizations of $n$. We also incroduce the two-point corrclation function $C$ of the medilan, assuming te fluctuations w be isetropic in the $x-y$ plane, and define it in such a way as to make the scaling of the problem clear:

$$
\left\{\mu\left(\dot{x}_{0}+\dot{x}\right) \mu\left(\dot{x}_{1}\right)\right\}=\left\{\mu^{2}\right\} C\left(\xi_{0}^{2} x^{2}\right),
$$

With this definition to is a meisure of the inverse seale length of the medium and $C$ decays on a unit scille length, c.g. $C(\tau)=\exp (-r)$. Later we will make sse of die ides of sp:stidl steraging and will alssume it io be identical to the ensemble averiging process i.c.

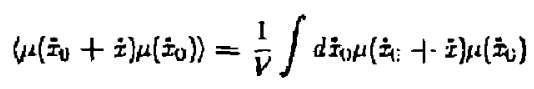


where $V$ is the two-dinensional volume of integration.

We then Fourier transform in the $z$ direction and consider a single $k_{2}$, i.c. a wave of the form $\exp (i t=z-i w t)$. The wave equation, (l), thus beciomes

$$
\nabla_{-\phi}^{2}-i \dot{A} \cdot \nabla \phi+k_{0}^{2}(1+\mu) \phi=0
$$

where

$$
\dot{A}=M \dot{z} \times \nabla \mu, \quad N=\frac{\left\langle\omega_{p c}^{2}\right\rangle}{\omega \Omega_{e}}, \quad k_{0}^{2}=\frac{\left\langle\omega_{j e e}^{2}\right\rangle_{j e}}{\omega^{2}} k_{e}^{2} .
$$

The quantity $M$ is obviously of great inportanes; in must cases of interest we expecs it to be larger than une, sonctimes much larger, making the convestive term in Eq. (3) die dominame scaticring term. The direct solution of Eq.(3) presenes considerable dificulties: in order to make progress we restrict ourselyes to cases in which the correlation length of the medium is large con.-pared to an incidese wavelength but smill cumpared to the scaucring length i.e. $k_{0} \gg \xi_{0} \gg 1 / l_{3}$ The first of these requirements: $k_{0} \gg \xi_{0}$ can be written $35\left(m_{i} i m_{t}\right) O_{l} N_{\| l}^{2} \gg \xi_{i l}^{2} \rho_{i}^{2}$ in terms of the usual tokamak parancters: $m_{1} / m_{e}$ is the mass ratio, $\beta_{4}$ the ion ben balue, $N_{\text {II }}$ the paralled index of refraction. and $\rho_{i}$ the ion l.ambur radius. These restrictions allow us to meglect interference effects and to treat the scallering process as a series of small, random perturbations which combine incohently to gise a diffusive process. The resulant diffusion cquation could be obuined by corefully going through a gesesal procedure to find the ware kinctic equation associated with Fq. (J). This approach is crertainly feasible, and was used by Oat ${ }^{4,5}$, and Sen and Fisch ${ }^{\text {t }}$ but it is perhups simpler to utitize the sirtilasity of Eq. (3) to the Schrocdinger cquation for a particle in a magnetic ficid:

$$
\nabla^{2} \Psi-2 \frac{i e}{h c} \dot{\mathcal{A}} \cdot \nabla \Psi+k_{i 0}^{2}\left[1-V-\left(\frac{e \dot{A}}{k_{0} h c}\right)^{2}\right] \Psi=0
$$

where $\dot{A}$ is the magnetic vector potential (in Coulumb gauge) and $V$ is the clectiostatic potental nomilised to the free energy of the particin: $h^{2} k_{0}^{2} / 2 m$. $m$ being the particle's mass. In addition $c$ is the speed of light. = the charge on the particle, and $h$ Planck's constant neer $2 \pi$. In the next section we "ill utijise the simitaricy of Fqs. (3) and (f). 


\section{THE WITESCATIERING LQLATION}

$\lambda$ cumpurison of equations (3) and (4) shows that our simplified lower hybrid wave equation is equivalent to Uhe Schroedinger cquation for a charged particle in a magnetic field $\dot{B}_{a}$ given by

$$
\dot{B}_{s}=M \nabla^{2} \mu \frac{h c}{2 e} \hat{z}
$$

and a nomalised potential

$$
v^{\prime}=-\frac{M^{2}(\nabla \mu)^{2}}{4 k_{0}^{2}}-\mu .
$$

The inequalities $k_{0} \gg$ to $_{1} \gg 1 /$, allow us to identify the energy density of the lower-hybrid wave whth the probability density of لye scaltered pafticles and thus achieve a difusion equation for the wave energy density? If the momentum of one particle is $\dot{p}$ with an average r.m.s momentum pu then the equation of motion is

$$
\frac{d \dot{p}}{d t}=-\frac{p_{j}^{2} \nabla V}{2 m}+\frac{p_{0} M}{\pi k_{0}} \dot{p} \times \hat{z} \nabla^{2} \mu
$$

The restriction that there be little scattering in a corrclation icngth thus requires $M^{2}\left(E_{0} / k_{0}\right)^{2}\left(\mu^{2}\right\rangle \ll 1$ so that to first order in the seattering we can atways take $V \approx-\mu$. Therefore if 42 follow a particle with initial momentum $p o \hat{p}$ we can write an expression for the rate of angular deflection

$$
\frac{1}{p_{0}} \frac{d \bar{p}}{d \tau} \approx \frac{1}{2} \nabla_{\mu}+M \hat{p}_{0} \times \frac{\nabla^{2}}{2} \frac{\nabla^{2}}{2 k_{0}}
$$

where we have laken the defiection to be small and the $z$ axis to be along $\hat{p}_{0}$.

We can thus write an expression for the mean squared angular deflection in a length $\Delta x$ which is small compared to the scarecring length but long compared to the correlation length,

$$
\left\langle\Delta \theta^{2}\right\rangle=\frac{1}{p_{0}^{2}} \int_{0}^{\Delta x} \int_{0}^{\Delta x}\left\langle\frac{d \dot{p}\left(x_{1}\right)}{d x_{1}} \cdot \frac{d \dot{p}\left(x_{2}\right)}{d x_{2}}\right\rangle d x_{1} d x_{2} .
$$

We proced by using $\dot{r}=\dot{x}_{1}-\dot{x}_{2}$ and replacing the ensemble avcrage by a spatial one so that the previous expression becomes

$$
\begin{gathered}
\left\langle\Delta \theta^{2}\right\rangle \approx \frac{\Delta x}{4 V} \int_{-\infty}^{\infty} d r \int d \dot{u}\left[\nabla \mu(\dot{u}) \cdot \nabla \mu(\dot{u}+\dot{r})+\left(M / h_{0}\right)^{2} \nabla^{2} \mu(\dot{u}) \nabla^{2} \mu(\dot{u}+\dot{r})\right] \\
+\frac{\Delta x}{4 V} \int_{-\infty}^{\infty} d r \int d \dot{u}\left[\nabla \mu(\dot{u}) \cdot\left(M / k_{0}\right) \hat{p}_{0} \times \dot{s} \nabla^{2} \mu(\dot{u}+\dot{r})\right. \\
\left.\quad+\nabla \mu(\dot{u}+\dot{j}) \cdot\left(M / \hat{u}_{u}\right) \hat{p}_{v} \times \hat{\Sigma} \nabla^{2} \mu(\dot{u})\right]
\end{gathered}
$$


Thıs cquation can be greotily simplificd by a little manipulation, e.g.

$$
\begin{aligned}
& \int d \dot{u} \nabla \mu(\dot{u}) \cdot \nabla \mu(\vec{u}+\dot{r})=\nabla_{r} \cdot \int d \dot{u} \nabla \mu(\vec{u}) \mu(\dot{u}+\dot{r}) \\
= & -\nabla \cdot \int d \dot{u} \nabla_{r} \mu(\dot{u}-\dot{r}) \mu(\dot{u})=-\nabla^{2} \int d \dot{u} \mu(\vec{u}) \mu(\dot{u}+\dot{r}) .
\end{aligned}
$$

Minipulating all of the terms in this manner and utifizing the definition of the two-pesint correlation function gives;

$$
\left\langle\Delta \theta^{2}\right\rangle=\frac{1}{4} \Delta x\left\langle\mu^{2}\right\rangle \int_{-\infty}^{\infty}\left[-\nabla^{2} C\left(\xi_{0}^{2} r^{2}\right)+\left(M^{2} / k_{\mathrm{v}}^{2}\right) \nabla^{\cdot} C\left(\xi_{\mathrm{v}}^{2} r^{2}\right)\right] d r
$$

u id: no cuntribution from the cross icrms.

The Fokker-Planck cquation for the particle distribution function, or cquivalently the lower-hybrid w are kinctic equaliun, is thus

$$
\frac{\partial F}{\partial t}+\frac{\omega}{\omega_{0}} \cos \theta \frac{\partial F}{\partial x}=D \frac{\partial^{2} F}{\partial 0^{2}}
$$

After interation by parts the diffusion coefficient $D$ is given by:

$$
D=\left\langle\mu^{2}\right\rangle \frac{\omega \xi_{0}}{4 k_{0}}\left[-\int_{-\infty}^{\infty} d x \frac{d C\left(x^{2}\right)}{d\left(x^{2}\right)}+6 M^{2}\left(\frac{\xi_{0}}{k_{0}}\right)^{2} \int_{-\infty}^{\infty} d x\left(\frac{c}{d\left(x^{2}\right)}\right)^{2} C\left(x^{2}\right)\right] .
$$

When $C(r)=\exp (-\tau)$ Fq. (12) becomes

$$
D=\omega \frac{\sqrt{\pi}}{4}\left\langle\mu^{2}\right\rangle \frac{\xi_{0}}{k_{0}}\left[1+6 M^{2}\left(\frac{\xi_{0}}{k_{0}}\right)^{2}\right]
$$

If ue restrict oursclues to time independent solutions and define an inverse scattering leagh $~_{g^{-1}}$ as $b_{j} D$ then we have the two-way diffusion equation

$$
\cos \theta \frac{\partial F}{\partial x}=\frac{1}{1} \frac{\partial^{2} F}{\partial \partial^{2}}
$$

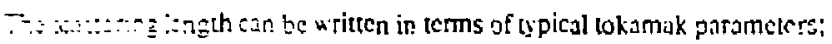

$$
!^{-1}=\frac{\sqrt{\pi}}{4} \xi_{0}\left\langle\mu^{2}\right\rangle\left(1+\frac{6 \rho_{i}^{2} \xi_{0}^{2} n_{0}}{N_{\|}^{2} r_{\mu}}\right)
$$

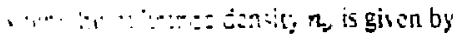

$$
==\frac{2 \nu^{2} T_{i} \cdot m_{i}}{4 \pi e^{2} e^{2} m_{e}}=8.9 \cdot 10^{9} \mathrm{~cm}^{-1 .}\left(\frac{T_{i}}{100 \mathrm{cv}}\right)\left(\frac{\nu}{1 G I_{2}}\right)^{2} .
$$


The relatively low value of this reference density makes it clear that that the derivative-coupling entm will dominate the scattering process

This scaltcring problem could also be approached thrciugh wase firmalism, in particular the treatment of Ott is perfectly good and his original result agrees exactly with the result of this paper. Eq.(13). the numerical factors being identical when the factor of 2 difference in the definition of Eo is recognifed. Howcser there were two compensating algebraic errors in the original paper, one of these was removed in a following Erratum ${ }^{5}$ but the other remained. So, paradoxically, originally the conclusic: was cerrect while after the Erracum it no longer is. We now point out the second algebraic error in Otis original papert. In his $\lambda$ ppendix $C$ Ott makes a chunge of variables from $k_{\perp}^{\prime}$ to $(l, \beta)$ where $l=\left|\vec{k}_{\perp}^{\prime}-\vec{k}_{\perp}\right|$ and $\vec{k}_{\perp}^{\prime} \cdot \vec{k}_{\perp}=k_{\perp}^{\prime} k_{\perp} \cos \beta$. He then states that under this transformatinn

$$
\delta\left(\omega_{k}-\omega_{k}\right)=\delta\left(l-2 k_{\perp} \sin \beta / 2\right) /(v \sin \beta / 2) \text {. }
$$

However this is not true. That the correct transformation is not ubvious stems from the change of variables not being one to one. If we write $x=k_{\perp}-k_{\perp}$ then $\delta\left[\omega^{\prime \prime}:-w_{k^{\prime}}\right]=\delta(x) / v$ and we have $l^{2}=k_{\perp}^{2}+k_{\perp}{ }^{2}-2 k_{\perp} k_{\perp} \cos \beta$ which implics

$$
l^{2}-4 k_{\perp}^{2} \sin ^{2} \beta / 2=\left(k_{\perp}-k_{\perp}\right)\left(k_{\perp}-k_{\perp}-4 k_{\perp} \sin ^{2} \beta / 2\right)
$$

so that

$$
\delta\left[x\left(x-4 k_{\perp} \sin ^{2} \beta / 2\right)\right]=\delta\left(l^{2}-4 k_{\perp}^{2} \sin ^{2} \beta / 2\right)=\frac{\delta\left(l-2 k_{\perp} \sin \beta / 2\right)}{4 k_{\perp} \sin \beta / 2} .
$$

We can write this as;

$$
\delta(x)=\left[\delta\left(l-2 k_{\perp} \sin \beta / 2\right)-\delta\left(x-4 k_{\perp} \sin ^{2} \beta / 2\right) / \sin \beta / 2\right] \sin \beta / 2
$$

the final delta function in Eq.(18) results from the non reversibility of the tralsformation and must be ignored. Then finally we have

$$
\delta\left(\omega_{k}-\omega_{k^{\prime}}\right)=\delta\left(l-2 k_{-} \sin \beta / 2\right) \frac{\sin \beta / 2}{\eta}
$$

which differs frum liq.(17) by a factor of $\sin ^{2} \beta / 2$. Originally this factur exaclly cincelled the crrot from Whe Jacubian of the transformation which was removed in the Ersatums . Ilence the correctusess of the 
result in the original paper. The same result can be found by periorming ule integration in Appendix C without making a change of variables.

\section{SOLETRONOF THE BIFUSION EUUITION}

Fiven though we may know the value of the scattering Iength appearing in Eq.(14), in order to predict inportant decails of the scatcered waye (e.g. Itrow aluch of an incident wave will penetrate a scatiering (cgime of length $l$ ) we must know sumelining of the solutions of this cquation. We will assume that there is a scattering bluck of isngth l, within which the cliffusion rate is constant. and the unseattered wave is incident from the lef at $x=0$. Arbitrary boundary conditions cannot be imposed; Eq.(14) is a two-way diffusion equation and just as the one-way diffusion equation $\partial F / \partial x=\partial^{2} F / 0^{2}$ would be well posed in the same domain only when boundary conditions are applied at $x=0,-\pi \leq \theta \leq \pi$ so this equation is well posed anly when $F$ is given on the merrals $x=0,0 \in[-\pi / 2, \pi / 2]$ and $x=l, \theta \in[-\pi,-\pi / 2], \theta \in[\pi / 2, \pi]$. This rescriction har the satisfying result that we cun only specify waves entering the seaucring block.

In our cxamination of the correct solution to Eq.(14) we will clascly folluw the treatment of Fisch and Kruskal, ${ }^{9}$ for convenience considering only solutions even in $\theta$. The boundary conditions are taken to be; $x=l, F=F_{i}(0), \theta \in[-\pi / 2, \pi / 2]$ and $x=1, F=0 \theta \notin[-\pi / 2, \pi / 2\}$. For sirnplicicy we will change the independent warizble $x$ to $z=x /$, so that Fq.(14) becomes

$$
\cos \theta \frac{\partial F}{\partial z}=\frac{\partial^{2} F}{\partial \theta^{2}}
$$

Ther is one immediate solution of Eq.(20), the so-called linear or diffusion solution; $(z-\cos 0)$ but this is neither positive definite nor capable of mecting the boundary conditions. In order to obtain the complete solution we lonk for separable solutions of the forn $f(0) e^{-\lambda x}$ resulting in the cquation

$$
\frac{d^{2} f}{d \theta^{2}}+\lambda \cos \theta f=0 \text {. }
$$

In trenting this cquation we can borrow froun the theory of Mathicu's equation ${ }^{10}$

$$
\frac{d^{2} f}{d \theta^{2}}+(\alpha+\beta \cos 20) f=0
$$


so that we know Eq.(21) will have solutions sith $f(2 \pi+\theta)=f(\theta)$ for only a denumerable set of distinct cigen-yalues: $\lambda_{n},-\infty<n<\infty$ with $n=0$ curcsponding to the degencrate linear solution in this cose and $\lambda_{-n}=-\lambda_{n}$. The set of functions $f_{n}(0) . n>0$ are orthogonal to each other and to any constant un the incerval $[-\pi, \pi$ ( (u'ith weighting function $\operatorname{ces} \theta$ ) but. with the addition of the linerr solution, complete ${ }^{11}$ only on $[-\pi / 2, \pi / 2]$. Sitnilarty the functions $f_{-n}(\theta)$ are proportionial to $f_{n}(0-\pi)$ and are complete on the rest of the interval $[-\pi, \pi ;$. Hence the complece solution to the diffusion proticm is

$$
\begin{aligned}
F(x, 0)=A & -B(z-\cos \theta) \\
& +\sum_{n=1}^{\infty}\left\{a_{n} f_{n}(\theta) \exp \left(-\lambda_{n} z\right)+b_{n} f_{-n}(\theta) \exp \left[-\lambda_{n}\left(l / l_{n}-z\right)\right]\right\}
\end{aligned}
$$

the coefficients $A, B, a_{n}, b_{n}$ are chosen so as to bit the boundary conditions at $2=0,1$. As the eigenvalues come in pairs and wis are deding with eren eigen-functions oniy in calculating the eigen-functions we will consider only pusitive eigen-valucs $\left(\lambda_{n}=k_{n}^{2}\right)$ and positive values of 0 . It is known ${ }^{12}$ that an approximate value fur $\lambda_{n}$ is $0.43\left(\lambda_{n}+1\right)^{2}, n>0$, so that the $k_{n}$ quickly hecume large as $n$ increases. Therefore whils we may have to produce the first few eigen-functions by numerical integration of Eq.(21) we expect to be able to use a W.K.B. type treatment for the rest if we define $S(\theta)=$ $\int_{0}^{0} \sqrt{\sin t} d t$ then the W.K.B. solution has the form;

$$
\begin{aligned}
& \left.f_{n}(\theta)=\frac{\exp -k_{n} S(\pi / 2)}{(-\cos \theta)^{\frac{1}{4}}} \cosh \mid k_{n} S(\pi / 2)-k_{n} S(\theta-\pi / 2)\right\}, \\
& \\
& \frac{\pi}{2} \geq \theta-\frac{\pi}{2} 2 k_{n}^{-3} \\
& \left.f_{n}(\theta)=\sqrt{\pi} k_{n}^{-\frac{1}{d}} A_{i}\left((\theta-\pi / 2) k_{n}^{-3}\right\}, \quad \mid \theta-\pi / 2\right) \ll 1 \\
& f_{n}(\theta)=\frac{\sin \left[k_{n} S(\pi / 2-\theta)+\pi / 4\right]}{(\cos \theta)^{\frac{1}{3}}}, \quad \frac{\pi}{2} \geq \frac{\pi}{2}-\theta \gg k_{n}^{-2}
\end{aligned}
$$

and the cigen-values are given by $k_{n} S(\pi / 2)=\left(n+\frac{1}{4}\right) \pi$. The accuracy of these cigen-finnctions can be checked cither by comparison with numerically produced solutions or by verifying their orthegnonalisy to $\cos \theta$. In Fig.] une of these cigen-functions is displayed.

Having produced ill or the required cigen-funetions the problem remuins that while the $f_{n}(n>0)$ are complete on $[-\pi / 2, \pi / 2]$ they are not orhogonal on that inicrval. Hence the usual method of 
integrating out the cocificients in Fq.(22) cannol be used, The method of fieting the boundary conditions used was to make a least squarcs fit. for accuracy sampling the crror at many more points than the number of eigen-functions involved. In Fig.? the fit at the boundaries is shown, taking the incident encrgy to have a Guussian spectnm. Once the eoefficients are sut the solution is knewn in the interval $[0, \eta$ and in Fig. 3 we display the the solution for $l=10 l$, using 20 eigen-functions. The fit can be improved by taking more cigen-functions but uheir addition does not cliange the character of the solution. The two-way diffusion equation allows a discontinuity of slope at $\cos \theta=0$ leading to the wecurrence of a Gibbs phenomenon in the fiuing of the cigen-function. In comparing Fig. 3 to a siniilar figure in uhe paper by Fisch and Kruskal it should be noled that in the calculation of tieir results incorrect eigen-values were uscd so that the higher frequency cigen-functions do not decay as quickly as they should. Fig. 4 is similar to Fig. 3 but this time the inconving flux specerum is isotropic, as it might be after refection from the tokamak wall. In the next section we will look more elosely at he physical

\section{Y. AMPRONIMIATF. SOI.UTION}

Once the melhod of solution is recognized dhe gross characteristics of the solution can be predicted from very simple ressoning without recourse to numerical comp̧utations. Our procedure depends on solving a sce of four simultancous equations which we obtain by making some assumptions about the angular dependance of the transmitted and reftected fluxes. If we denote the transmitted fux by $T_{f}$ we can immediately wrie down one equation; as all of the Mathicu eigen-functions ate orthogonal to $\cos \theta$ on $[-\pi, \pi]$ the forward flux is given by

$$
T_{f}=B \int_{-\pi}^{\pi} \cos ^{2} \theta d \theta=\pi B
$$

Whenl is much greater than $l_{x}$ the transmitted flux spectrum will be independent of be incidint flux $\because \cdots !$ inm and uc choose to approximate is by $t \cos \theta$ (we cxamine the validity of this assinmpion hater). 1.w ding cur ewo salues for the transmiticd fux gives ur

$$
t=2 B
$$


The reinected flux spectrum. houeser, will be dffected by wie inciusne nux spectrum and though the cxact form of our approximation will not greatly affect our resuit we will treat separatsly the cases af peraked and isotropic spectra. When the incoming spectum is iso:ropic we take the reflected flux to be scotropic also and equal to $r$ : this gives us a criterion for fux cunsern ation of une form

$$
1=\frac{r t}{2}+2 r .
$$

In the case of a relatively narrow Gaussian incoming speclrum, as in our dird figute we take the reflected flux sicetrum to be $r \cos \theta$. Flux couseridion then gives

$$
I=\frac{\pi}{2}(l+s)
$$

We can derive two more equations in the lollowing manner. All of the Mithicu cigen-tunctions decay rapidly untside of their dumain of completeness hence at $x=1,0=0$ only the linsar solution wili: contribute giving $t \approx A-B(1 / l,-1)$. The same ressoning will apply at $x=0,0=\pi$ implying. $r \approx A-B$. We can solve the above eqtations in the rwo cases we have considered tu give firstly for an isotropic incoming spectrum, $B=1 /\left(\pi+2 / / l_{b}\right), A=\left(l / l_{0}+1\right) /\left(\pi+2 l / l_{s}\right), t=2 /\left(\pi+2 t / l_{s}\right)$ and $r=\left(l / l_{s}\right) i\left(\pi+2 l / l_{s}\right)$. The predicted value for the fiux uansmission cocfficient is thus

$$
T_{f}=\frac{\pi d_{1}}{2 I+\pi \tau_{\rho}} .
$$

When we have a peaked iicoming spectrum our equations give; $B=2 / \pi\left(l / l_{0}+2\right), A=2\left(l / l_{\mathrm{s}}+\right.$ $\left[j / \pi\left(l / l_{s}+2\right), t=4 / \pi(l / l,+2)\right.$ and $r=2(l / 6) / \pi(l / h,+2)$. In this cose the predicted $f_{3} \mathrm{x}$ transmission crefficient is

$$
T_{f}=\frac{2 l_{0}}{i+2 l_{0}}
$$

In Fig.6 the ratio of the predicted and numerically produced transmitted fiuxes are plutted for $/$ from D to $2 \boldsymbol{n}_{3}$. Considering the simplicity of the derivation the ageement is temarkably good. In figures 7 and 8 our asstimptions on the fonn of the tansmitted and reflected huxes are ryantined. For both the isotropic ard peaked cases we assumed that the uansnitued thux could be taken to have a cos 0 dependence. is $l / l_{4}$ increases. Figs. $7 \mathrm{~b}$ and $8 \mathrm{~b}$ show that the the trinsmitted fux ux spectrun rapidly

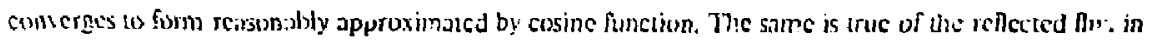




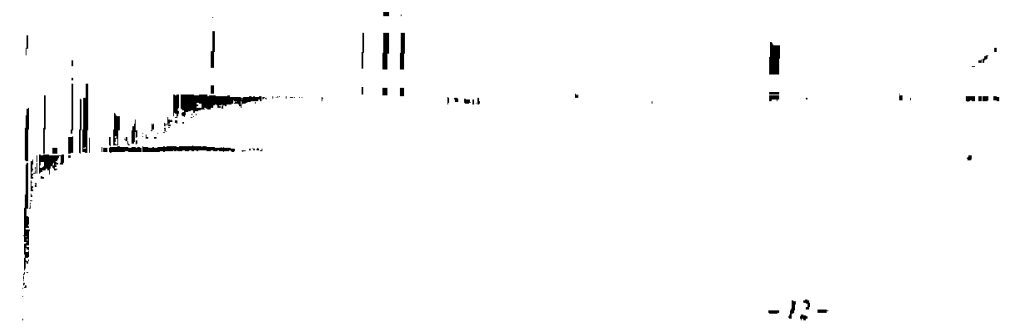

the peaked case as shown by Fig. 7a. As $/ /$, becomes large the retheced flux in the isotropic case renciles gond agreement with our assumed isutrupic reflettion spectrum ss secn in Fig. $8 \mathrm{~b}$.

\section{i. CONCILISIONS}

In this puper we have shoun a straightfontard method we derive the diffusion equation for the staltering of luwer lybrid wares, pointing out the critect salue of the diffusion coeflicient. We showed how this cquition could be sulned numerically, with the correct boundary conditions. Our insthod of solucion sugecented a simple model that was used to provide an analyzic expression for the flux trallsmission sodficient which agreid well with our numerical res dls. This model showed that for hirge $l / l$, the flux transmission cocficient taries inversely with $l$. The expansion in Mathieu sigen-functions malk es it clear that in the body of the scatcering nedium the lux density decays lincarly, white the ransmitted dux stectum rapid): luses its correlation with the incoming spectntm converging to a limiting form represchted nodessly well by a ansin: dependenec. Thete are seteral pussible cxicusions: a more realistic geomery suuld be used. a small amount of tine dependence could be introduced, or the diffusion constant could te allowed to slowly vary. Howerer it as wifficult to see how the

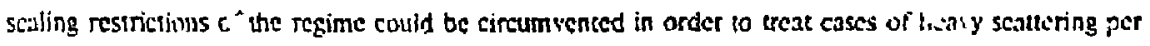
currelation length. In that regime interference effects cannot be neglected and numerical sulutions of the original wate equation are probably all that can be obtainod.

\section{.CKNOHLEDGMENTS}

One of us (P.I.A.) would like औhank Dr. N.J. F. sch for sescrul helpful discussions. This work was supported by the U.S. Department of Energy under Contract DE-ACD2-76-C1103073. 
Referencus

'T. it. Slix. Phys. Rev. Lett. 15. 878 (1965).

${ }^{2}$ C. '1. Surko and R. E. Siusher, Phys. Rer. I.ett. 37. 1747(1976).

${ }^{3}$ R. E. Sillusher and C. גi. Surto, Phy s. Rev. L.et. $40, \$ 00$ (1978).

${ }^{1}$ E. Ott ihys. Fluids 22. 17.12 (1979).

${ }^{5}$ E. Ot ?hys. Fuids $23,+21$ (1280).

${ }^{8}$ A. Sen and N. J. Fisch, hlassachusets Institutc of Technulugy Plasmd Rese:tr.h Report No. PRR 78/16 (1978).

'L. A. Chernov, Hare Propagarion in a Random M/rdism (M.LOraw-Hii!, New York. 1960).

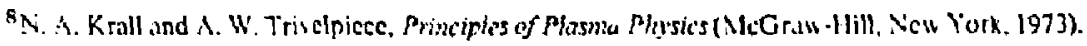

${ }^{9}$ N. J. Fich and .V. D. Kniskal. J. Math. Plıys. 21, 740 (1980)

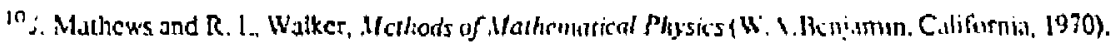

"R. Beal. Submittid to J. Wath. Phys.

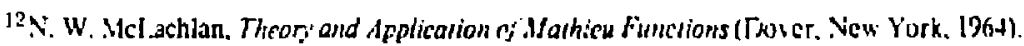




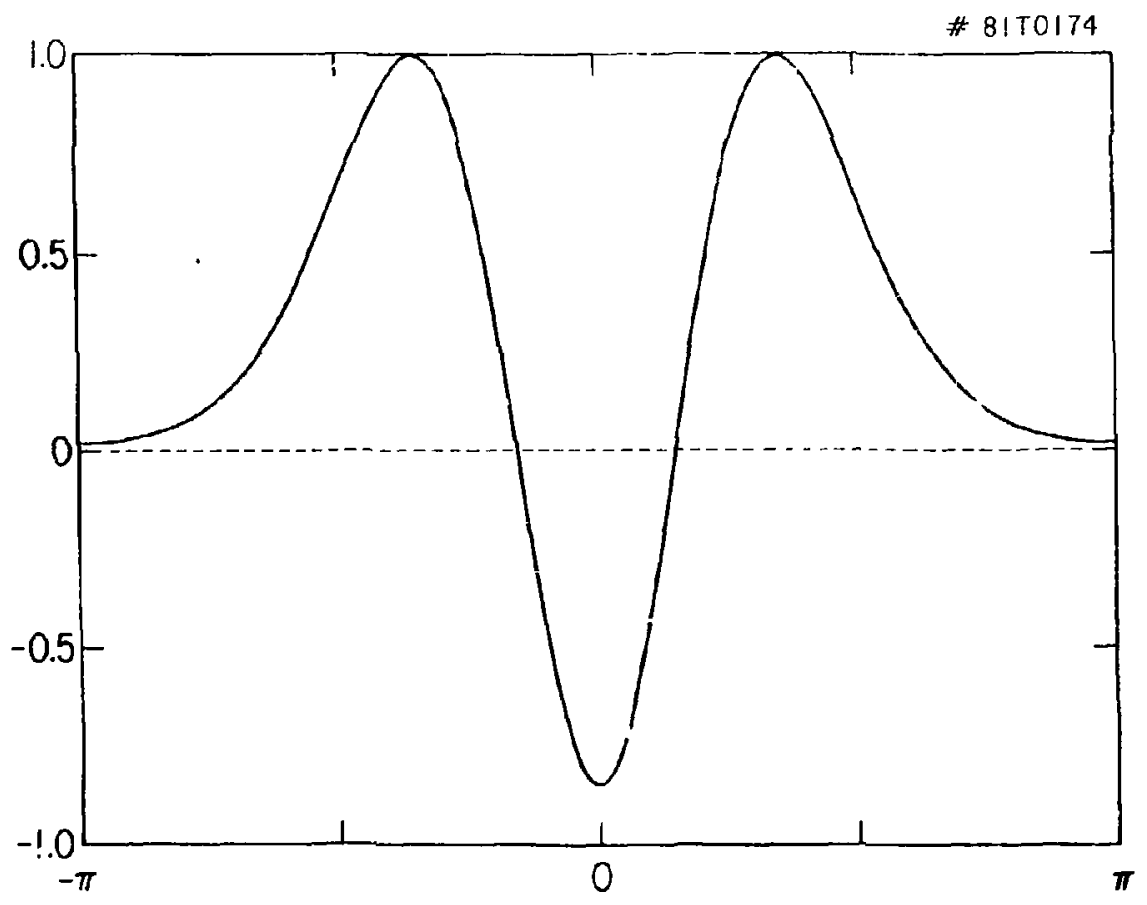

ro

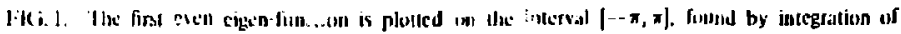

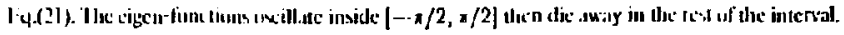


p. 15

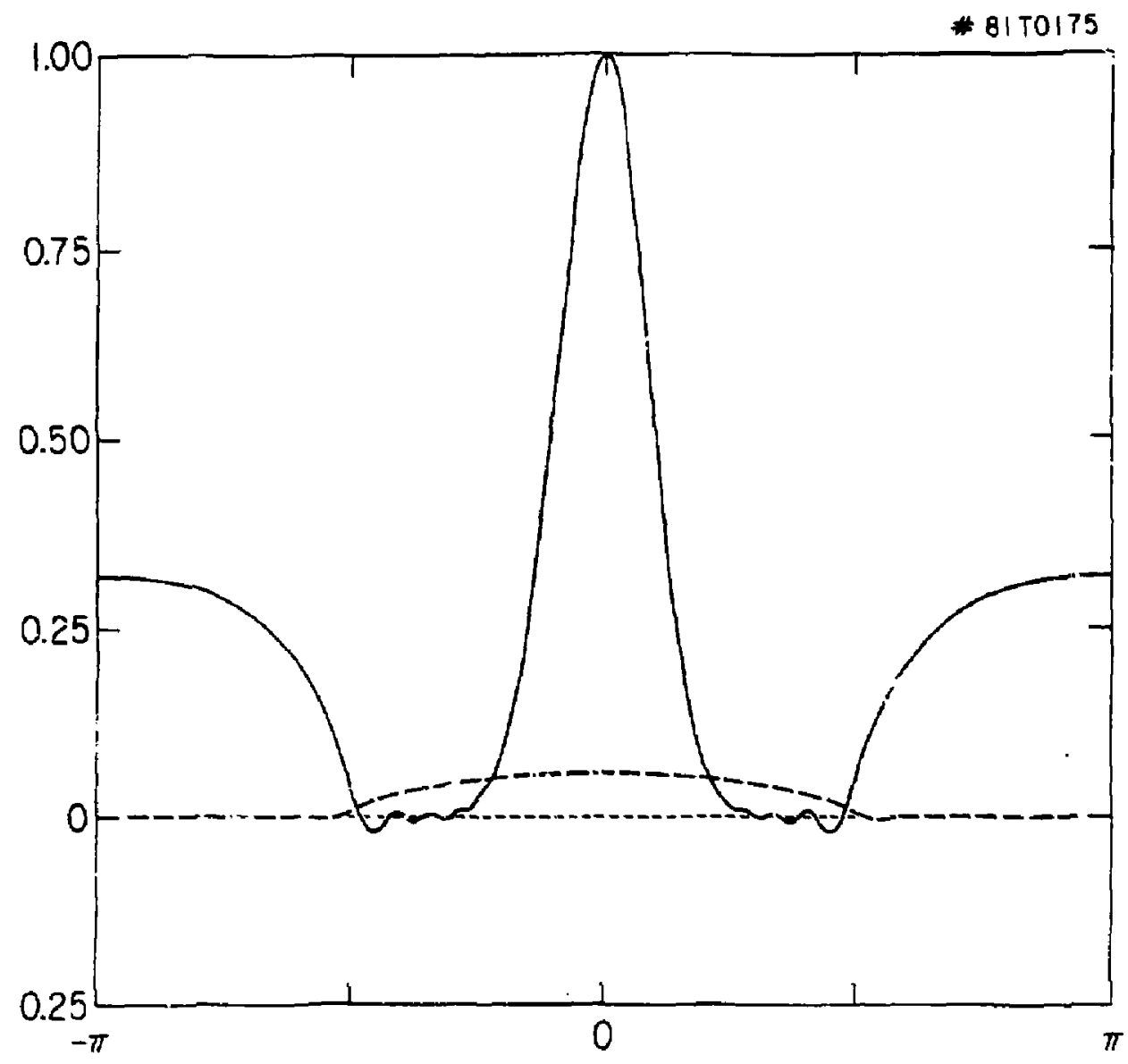

FIG. 2. The fit of the solution to the boundary conditions at $x=0$ (solid line) and at $x=1$ (dashed linel is shown usin- 20 cigen-functions and Gaussian incoming stecerum. 


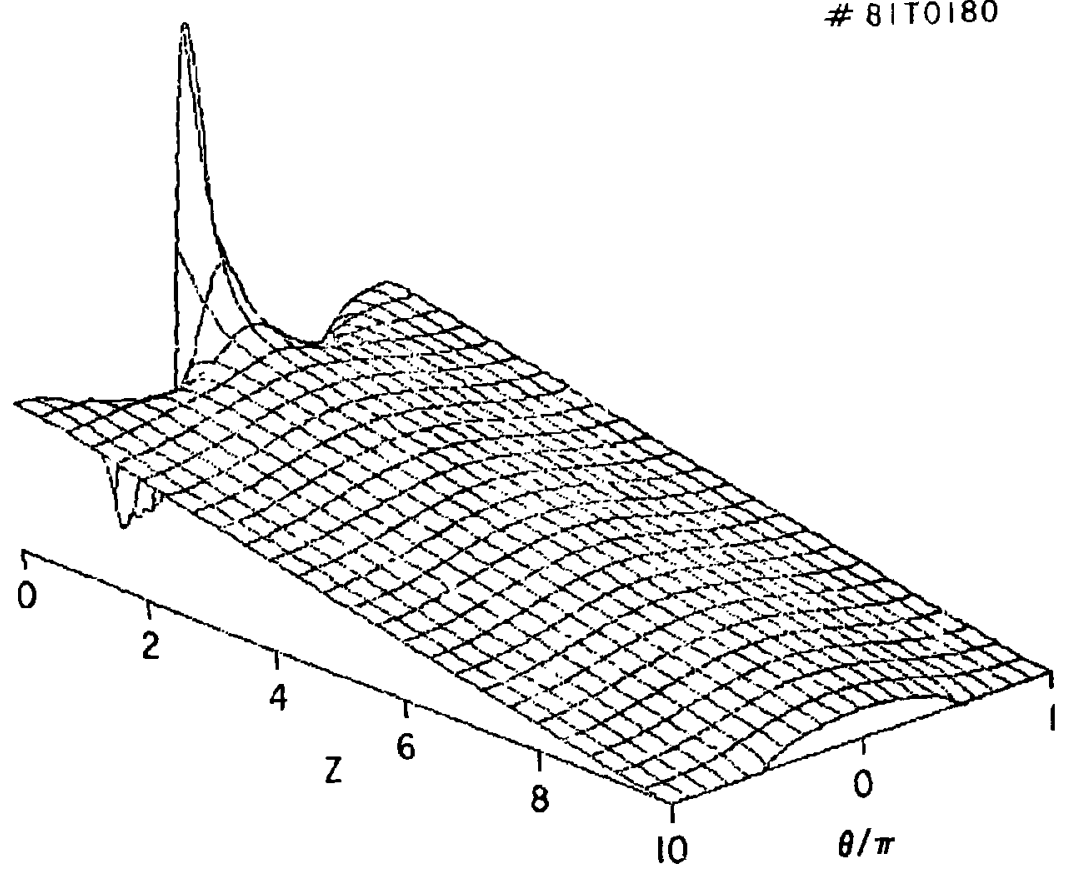

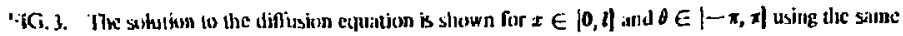
buundary cunditions as in Jïg.2. 


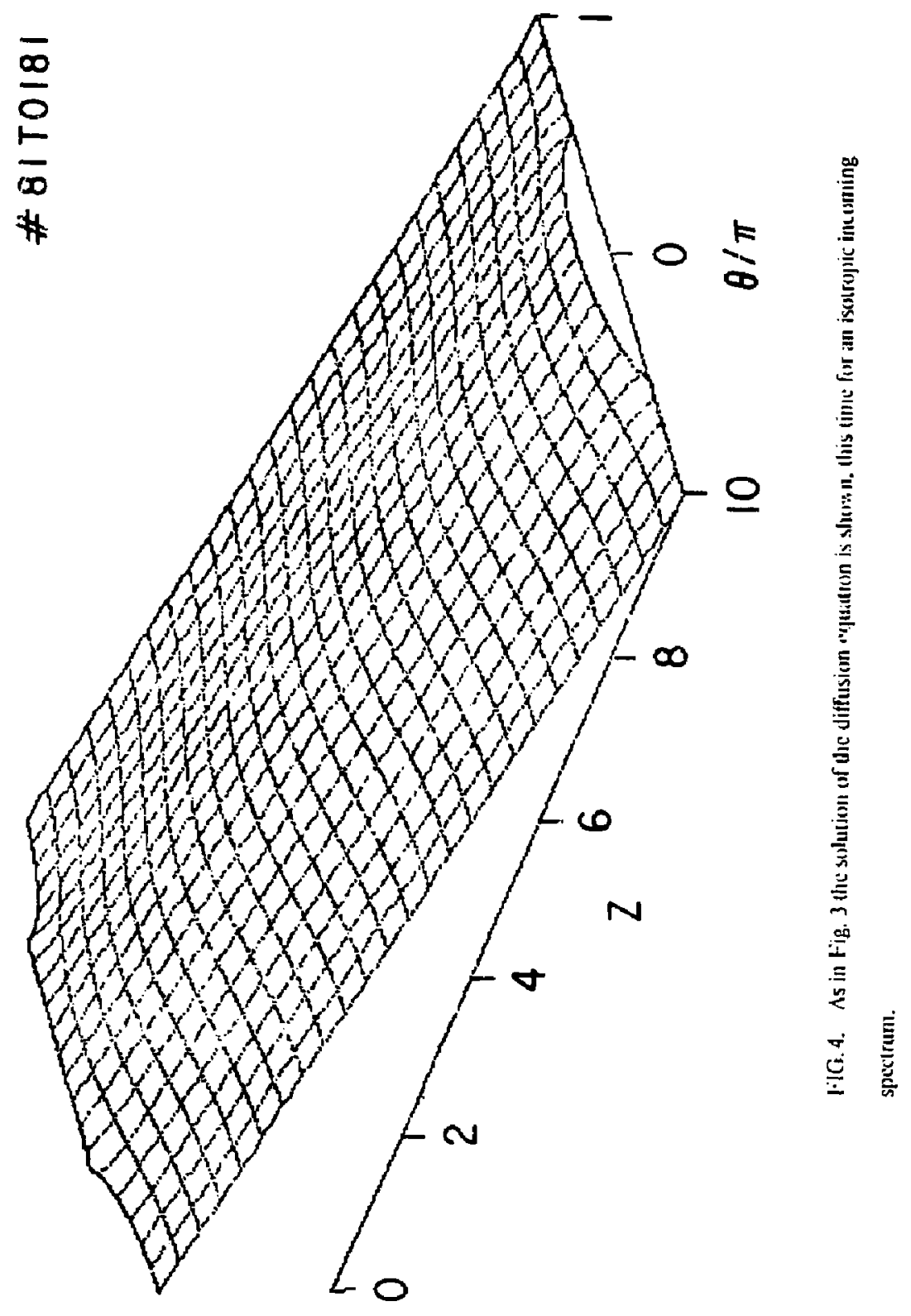





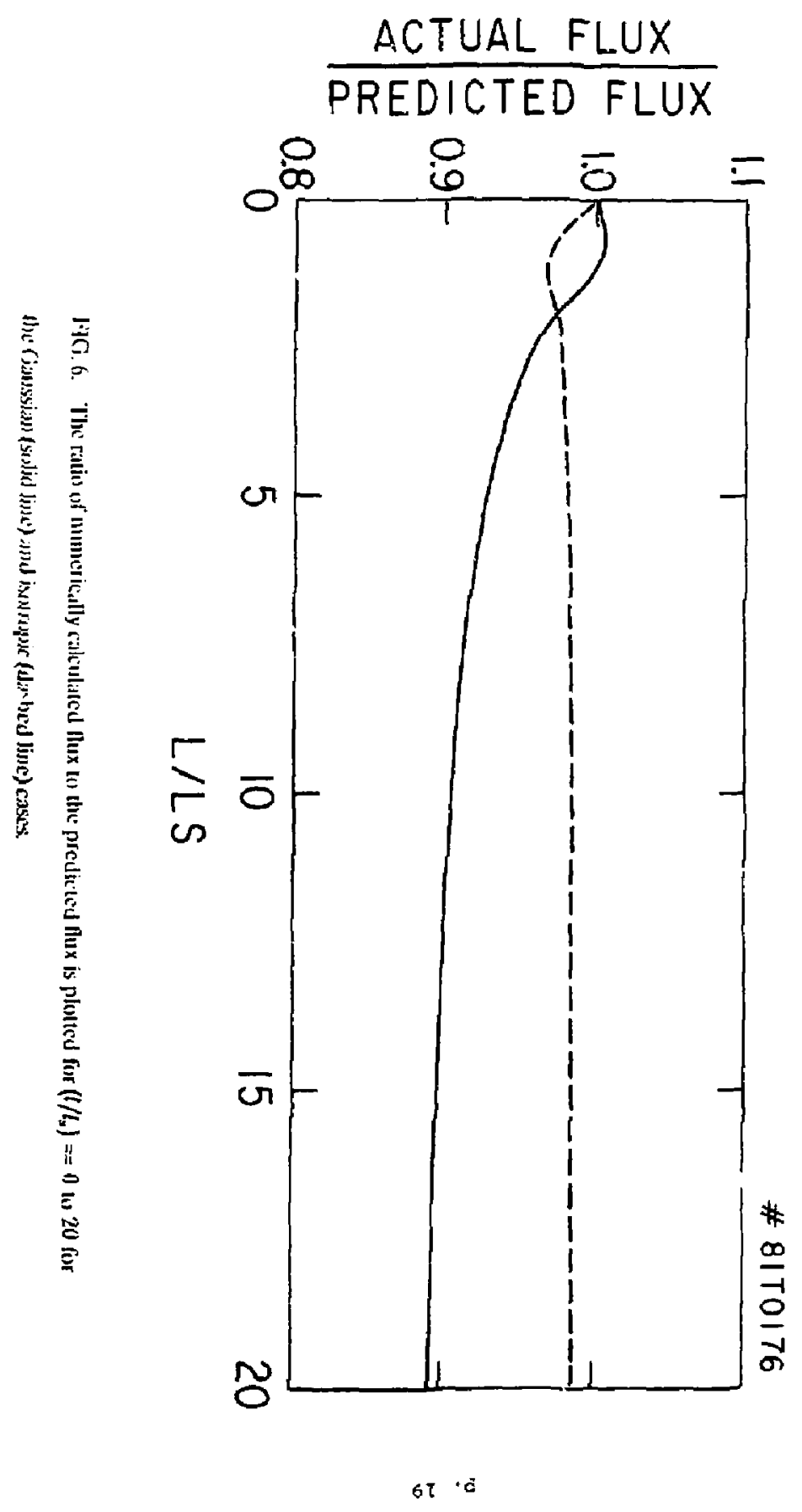




$$
\text { 上. } 20
$$
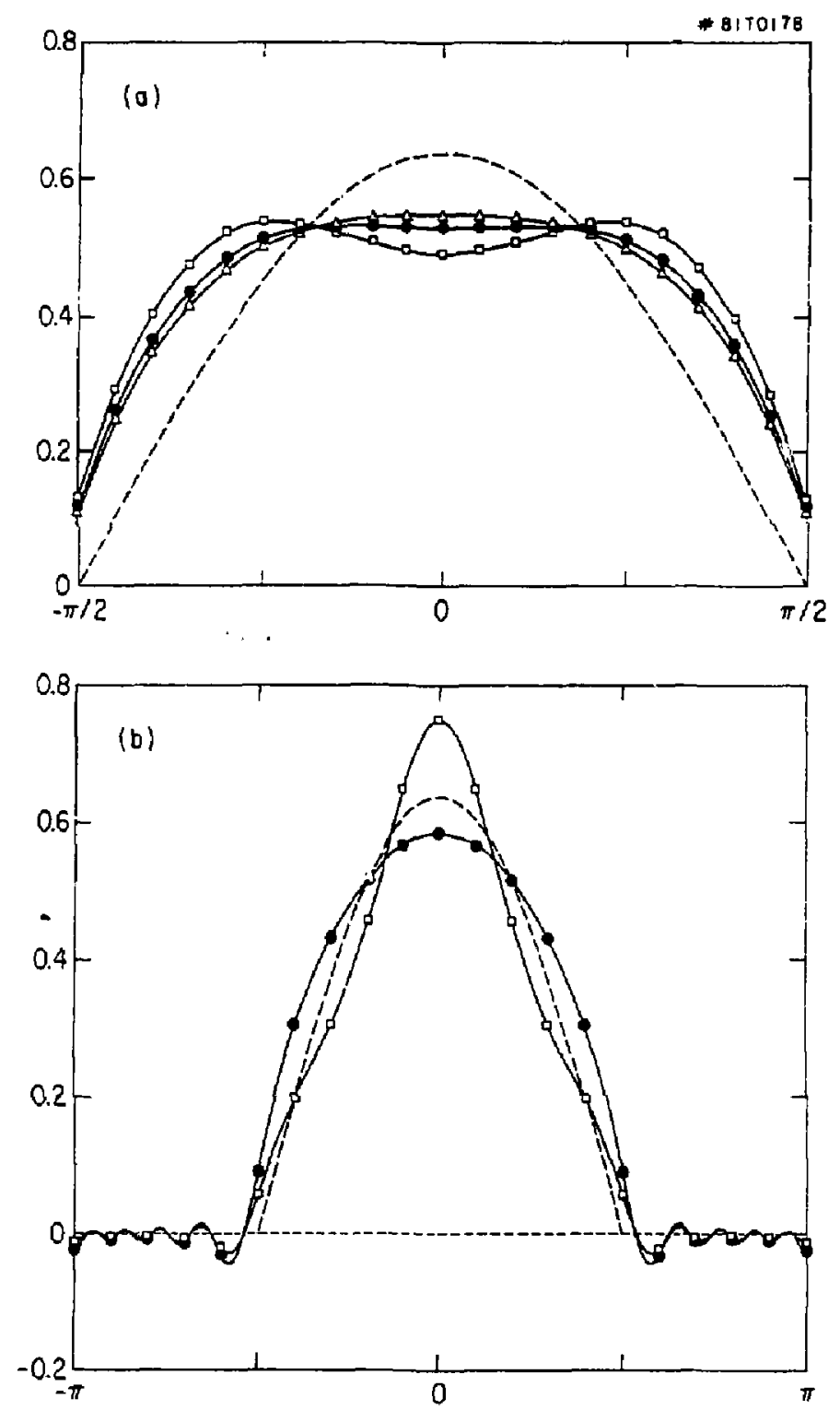

FJG. 7. The reflected (figure a) and transmitted (figure b) flux spectrum (both nomalized to unity) from a Garssian incoming spectruen are displayed for $l / l_{s}=0.5,2.5$ and 10 . The dashed line is the correspor.ding nomnalized cosinc approximation. 
p. 21
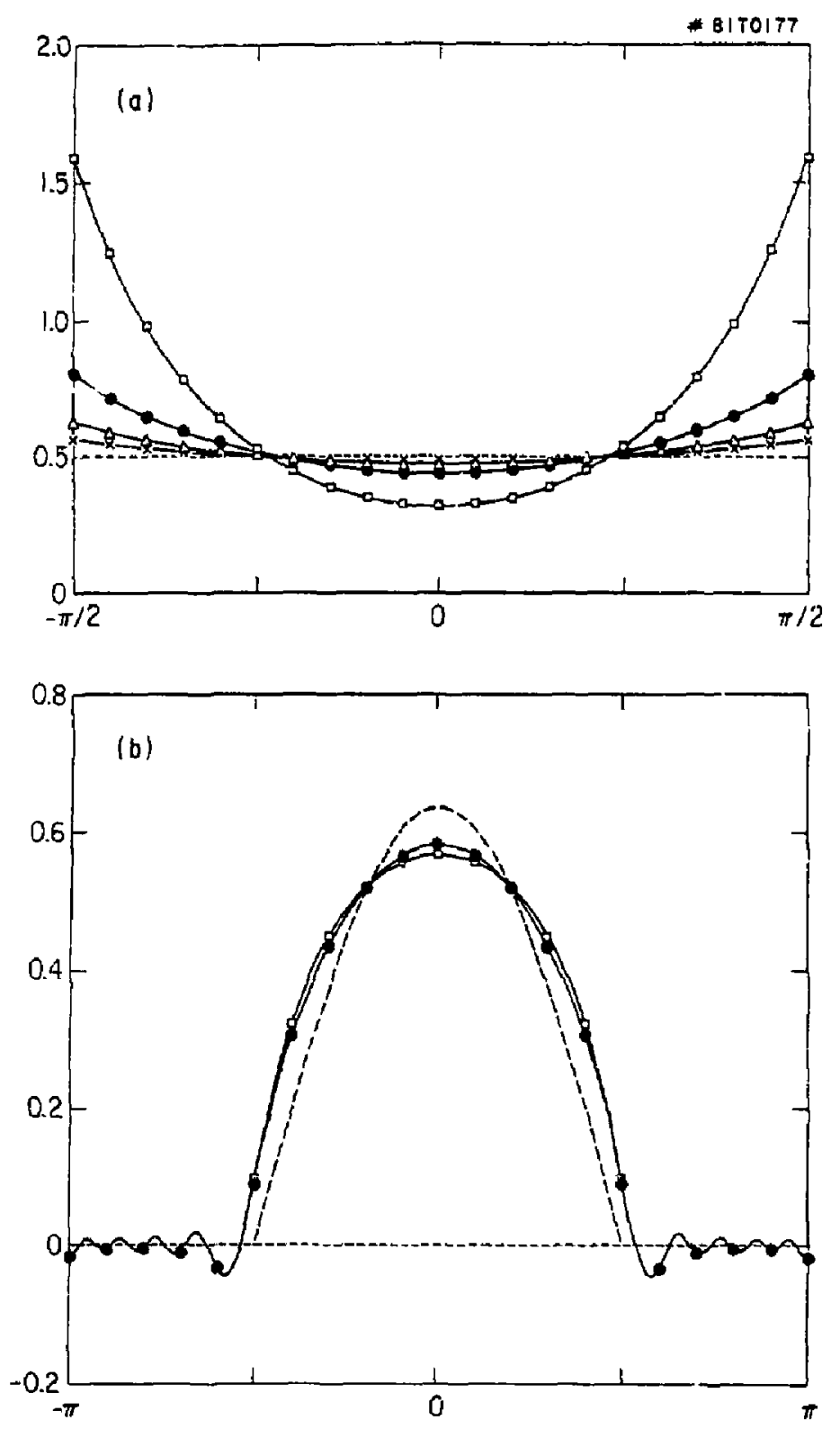

FIG. 8. The same information as in Fig. 7 is plotted for an isotropic incoming spectrum with the corresponding approximation shown as a dashed line. 